\title{
Development and Evaluation of a Powered Artificial Gastrocnemius for Transtibial Amputee Gait
}

\author{
Michael F. Eilenberg (D), Jiun-Yih Kuan (D), and Hugh Herr (D) \\ Biomechatronics Group, MIT Media Lab, Massachusetts Institute of Technology, Cambridge, MA, USA \\ Correspondence should be addressed to Hugh Herr; hherr@media.mit.edu
}

Received 15 April 2017; Revised 30 August 2017; Accepted 25 October 2017; Published 22 January 2018

Academic Editor: Kazuo Kiguchi

Copyright (C) 2018 Michael F. Eilenberg et al. This is an open access article distributed under the Creative Commons Attribution License, which permits unrestricted use, distribution, and reproduction in any medium, provided the original work is properly cited.

Existing robotic transtibial prostheses provide only ankle joint actuation and do not restore biarticular function of the gastrocnemius muscle. This paper presents the first powered biarticular transtibial prosthesis, which is a combination of a commercial powered ankle-foot prosthesis and a motorized robotic knee orthosis. The orthosis is controlled to emulate the human gastrocnemius based on neuromuscular models of matched nonamputees. Together with the ankle-foot prosthesis, the devices provide biarticular actuation. We evaluate differences between this biarticular condition and a monoarticular condition with the orthosis behaving as a free-joint. Six participants with transtibial amputation walk with the prosthesis on a treadmill while motion, force, and metabolic data are collected and analyzed for differences between conditions. The biarticular prosthesis reduces affectedside biological knee flexion moment impulse and hip positive work during late-stance knee flexion, compared to the monoarticular condition. The data do not support our hypothesis that metabolism decreases for all participants, but some participants demonstrate large metabolic reductions with the biarticular condition. These preliminary results suggest that a powered artificial gastrocnemius may be capable of providing large metabolic reductions compared to a monoarticular prosthesis, but further study is warranted to determine an appropriate controller for achieving more consistent metabolic benefits.

\section{Introduction}

For those living with transtibial (below-the-knee) amputation, prosthetic technology has yet to fully restore the functionality of the missing biological structures. As evidence of this technological limitation, transtibial amputees wearing conventional prostheses naturally select slower walking speeds than those with biological limbs, and, when walking at the same pace as nonamputees, they require more metabolic power than their nonamputee counterparts [1-4]. Further, transtibial amputees exhibit kinetic pathologies, particularly during the late-stance knee flexion phase of gait, where the knee flexes to prepare for the swing phase. Transtibial amputees walking with conventional prostheses increase their affected-side hip positive and negative power during this phase, compared to nonamputees, possibly in response to the lack of power from the calf muscles [4].

Unlike conventional prostheses, new robotic powered ankle-foot prostheses (PAFP) can output net mechanical work similar to that of the human ankle-foot complex.
Consequently, amputees using powered prostheses have preferred walking speed, metabolic cost at a given speed, and contralateral limb impacts that are not significantly different from those of nonamputees $[5,6]$.

These robotic prostheses, however, still act only at the ankle joint and hence can only provide monoarticular ankle function. In contrast, the gastrocnemius calf muscle both plantar flexes the ankle and flexes the knee. Hence, amputees walking with monoarticular prostheses must compensate for this lack of function by increasing activation of the remaining knee flexors in the hamstring group [7-9]. This increase in activation is still seen with robotic ankle-foot prostheses [7]. Therefore, it is likely that biarticular interventions are required to provide a more complete restoration of the missing musculature.

Only few studies have been done to evaluate how a biarticular robotic device could improve metrics of gait for transtibial amputees beyond monoarticular devices. In the first such study, researchers approximated the gastrocnemius muscle as isometric during walking and built a 
clutched-spring joint into a knee orthosis, called an artificial gastrocnemius (AG), to replicate the modeled function of an isometric muscle in series with a compliant tendon [10]. This AG-PAFP combination was shown to reduce metabolic cost of a bilateral transtibial amputee, as compared to a conventional passive transtibial prosthesis.

However, this previous study was limited in that it did not evaluate the individual contributions of the PAFP and AG on metabolism. Hence, it is not known whether the metabolic effects stemmed from the actions of the PAFP, AG, or both devices. Researchers have since attempted to isolate the incremental influence of the AG on metabolism and kinetic measures by comparing an AG-PAFP combination to a PAFP-only condition with identical mass distribution. This study provided preliminary evidence that the inclusion of the AG may, in fact, improve metabolism and influence several kinetic measures, compared to the PAFP-only condition [11].

Still, these two aforementioned studies only considered isometric behavior of the biological gastrocnemius muscle. In contrast, the human gastrocnemius muscle is responsible for 3-5 Joules of positive net mechanical work per step [12, 13]. This positive net work is impossible to reproduce with a quasi-passive device, yet its presence may be critical to the advancement of active transtibial prostheses of greater efficacy.

For the present study, we develop a novel AG that can provide positive net mechanical work. We develop a controller, based on a neuromuscular model, for said device that emulates the functionality of the missing gastrocnemius. We also develop a torque controller to enforce this functionality. We anticipate that the applied moments and positive net work from the AG will replace affected-side biological knee positive work and flexion moments. Further, we expect that the AG's improvement of calf muscle function will reduce the late-stance pathologies typically seen in the affectedside hip [4]. Therefore, we hypothesize that the use of this AG, in conjunction with a PAFP, will reduce both biological flexion moment impulse and positive mechanical work of the affected-side knee and hip joints during late-stance knee flexion, as compared to the same conditions with only the PAFP. We further hypothesize that these changes will provide a corresponding reduction in metabolic cost of walking via the reduction in metabolically expensive positive muscle work.

\section{Powered Biarticular Transtibial Prosthesis Design}

It has been shown that adding mass to the lower extremities can have a detrimental effect on walking metabolism [14]. Hence, the goal in this project was to provide interventions to a participant walking on a treadmill by remote actuation, so to minimize mass affixed to the wearer. To this end, we developed a cable-driven tethered exoskeleton system, comprising a motor drive module, a cable transmission linkage, a robotic knee joint, and a custom knee orthosis. We first describe the mechatronic design of these four elements and then describe the torque control of the system in the next section.

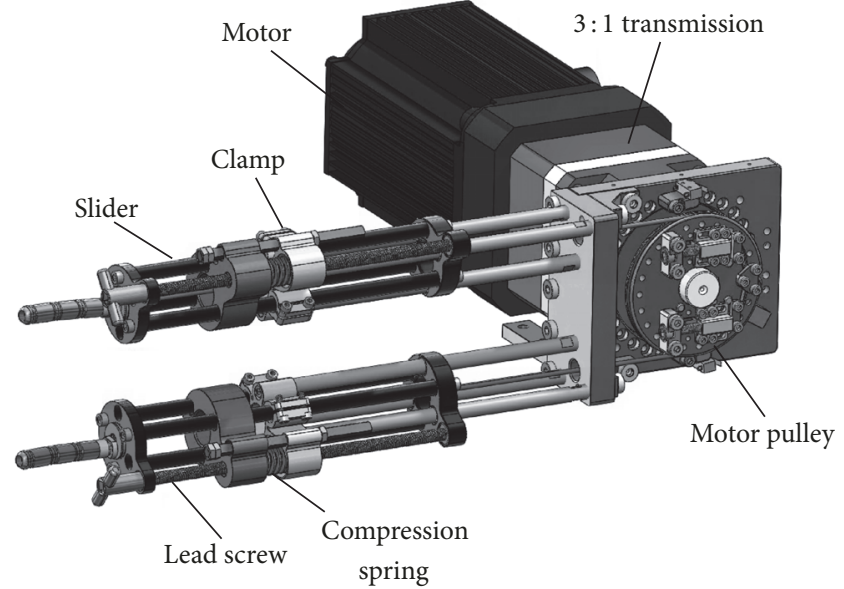

FIGURE 1: Motor drive module: actuation source. The tensioner attached to the motor drive unit and provided the ability to preload the cables to a desired tension without adding series compliance to the system.

2.1. Motor Drive Module. The core of the motor module was a powerful offboard actuation source and real-time sensing and control system. The actuation source included a $3 \mathrm{~kW}$ AC servo motor (SGMSV-30A3A61, YASKAWA), a $3: 1$ gear reducer (042PLX0030-LB-04027, CGI INC), and a custom cable tensioner. The sensing and control system, designed for real-time control and system safety, included a host personal computer, an EtherCAT ${ }^{\circledR}$ master controller (SPiiPlusNTM, ACS Motion Control), local servo controllers (V200AE1A002000200, Yaskawa), and an EtherCAT ${ }^{\circledR}$ slave I/O system (750-534 EtherCAT Coupler, WAGO). This system had multiple analog and digital inputs that were used for gathering feedback signals in real-time from the device for the purposes of data collection and feedback control. A $90 \mathrm{~mm}$ diameter pulley was mounted to the transmission output shaft to actuate the drive cable.

A spring-based tensioner system was used to preload the cable. This tensioner was a bracket with an internal slider, driven by a lead screw (Figure 1). This joint enabled the bracket to expand to take up cable slack. A spring was placed in series between the sliding element of the bracket and the stationary element, serving as an indicator of cable preload. As the lead screw was manually rotated, the bracket expanded and provided tension on the cable. The reaction force from the cable conduit was transmitted through the bracket and loaded the spring. Therefore, by measuring the spring deflection during the cable preloading, the cable tension could be approximated. Once the cable was pretensioned, screws were tightened to lock the slider to make the tensioner a rigid structure. Therefore, the tensioning springs did not introduce series compliance to the system during operation of the cable drive.

2.2. Cable Linkage. The cable linkage consisted of an inner cable, flexible conduits, rigid tubing, and a pulley system. A $3 \mathrm{~mm}$ diameter $7 \times 19$ braided steel inner cable was used to transmit power from the motor to the knee joint pulley 


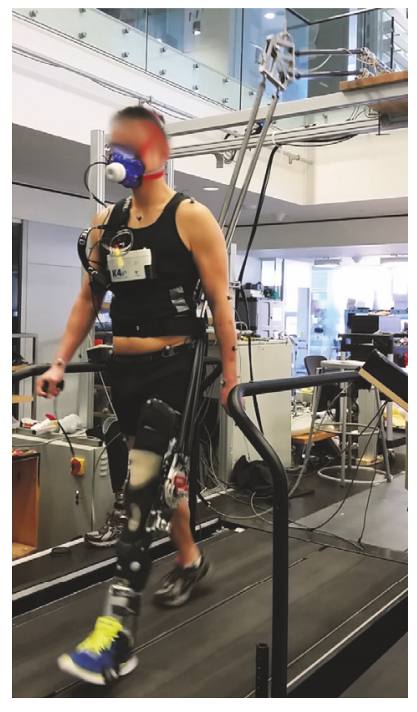

(a)

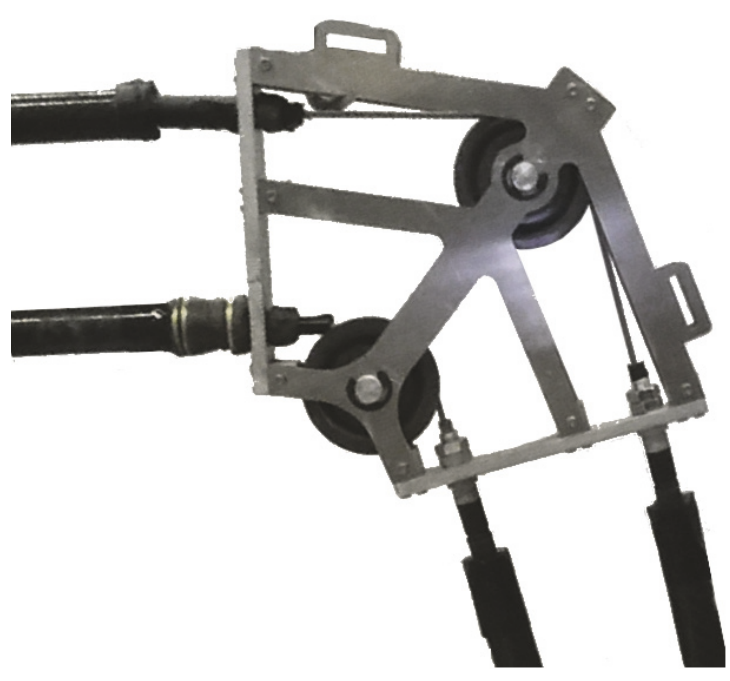

(b)

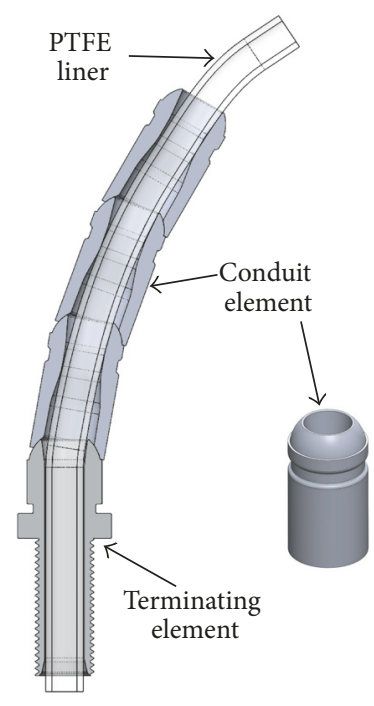

(c)

FIGURE 2: Conduit linkage as it interfaces with the artificial gastrocnemius and participant. The complete biarticular prosthesis system is shown (a) along with close-up views of the conduit linkage pulley system (b) and components of the flexible conduit sections (c).

in a similar way as a Bowden cable drive. However, instead of a flexible conduit throughout the entire tether, sections of rigid tubing and a pulley system were used (Figure 2). The tubing was made of $0 / 90$ carbon fiber, with $22.2 \mathrm{~mm}$ outer diameter and $19.1 \mathrm{~mm}$ inner diameter. This system had a distinct advantage over a traditional, flexible Bowden cable drive: the losses due to friction between the cable and conduit were near-zero through the tubing sections, as the cable did not contact the sides of the conduit.

Short, flexible conduit sections were used to link the straight rigid tubing together in order to provide articulation. Typical Bowden cable conduits have the tendency to straighten when tension is applied to the cable. Rather than using such a conduit, short, interlocking aluminum conduit elements were designed (Figure 2(c)). These elements were designed in case the length of the inner bore of the conduit did not change upon bending. Each element had a male and female end, that, when mated, produced a ball-and-socket joint, through which the cable could pass. These elements were strung together in series to produce flexible conduit sections of the desired lengths. Polytetrafluoroethylene (PTFE) liners ( $3 \mathrm{~mm}$ inner diameter, $6 \mathrm{~mm}$ outer diameter) provided low-friction interface within these sections of conduit elements.

The conduit linkage was arranged behind and above the treadmill, so to provide minimal interference to a wearer of the knee joint (Figure 2(a)). The conduit linkage allowed two degrees of freedom in the sagittal plane, via the vertical and horizontal hinging of the conduit sections. Some limited motion in the coronal and transverse planes was also possible with this system. The remaining flexibility needed near the waist of a human subject was provided by a section of several conduit elements linked in series, forming a flexible section of conduit.

The added mass from this conduit system was expected to provide some downward force to the wearer during walking.
This force was estimated by using a force gauge to hold up the AG with the conduit linkage at the location where a typical participant's knee would be during walking.

2.3. Robotic Knee Joint. The actuated robotic knee consisted of a pulley, driven by the tether cable, which sat lateral to the wearer's affected-side biological knee joint (Figure 3). This pulley was free to rotate on ball bearings in the sagittal plane about the knee center with respect to the joint housing. The pulley itself was connected to the output link through a torsional spring (stiffness $=1200 \mathrm{Nm} / \mathrm{rad}$ ), which acted as a series compliance between the cable drive and the output link.

A rotary potentiometer $(8 \mathrm{kOhm})$, mounted between the joint housing and the output link, was used to measure joint angle. This potentiometer was mounted on the lateral side of the joint, with the potentiometer shaft going through the center of the joint and attaching to the output link on the medial side (Figure 3). Torque sensing was accomplished via the use of a full-bridge strain gauge (SGT-2/350-FB13, Omega Engineering, Inc.), mounted on the output link so that bending of the link in the sagittal plane would be detected by the strain gauge. The strain gauge was connected to a custom preamplifier that included a common-mode input filter to reject electromagnetic interference and a secondorder low-pass filter with a cutoff frequency of $585 \mathrm{~Hz}$.

The designed robotic joint had physical parameters within the desired values (see Table 1). Although the robotic joint itself had a mass of only $0.6 \mathrm{~kg}$, the orthosis for attaching it to the body required the addition of $0.8 \mathrm{~kg}$ and $1.3 \mathrm{~kg}$, respectively, for the amputee and nonamputee subjects. In addition, the suspended cable linkage provided approximately a $0.6 \mathrm{kgf}$ downwards force at the knee. Regarding cable pretension, it was found that $30-50 \mathrm{~N}$ of cable pretension kept cable friction low without introducing bandwidth-limiting cable slack. 


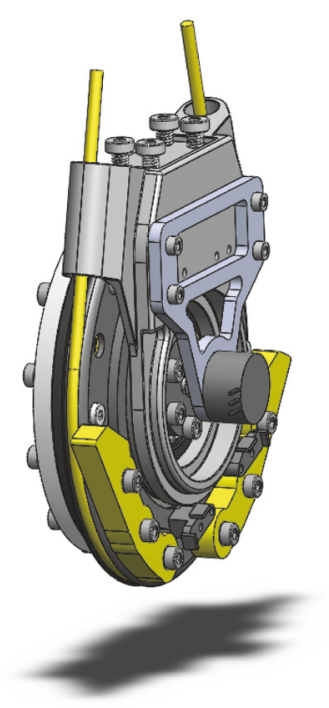

(a)

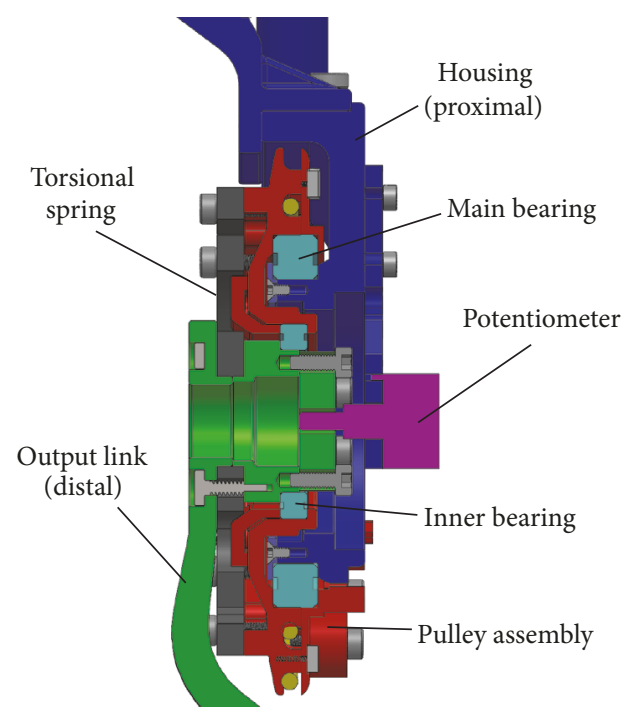

(b)

FIGURE 3: Powered artificial gastrocnemius joint. The assembled joint is shown without proximal and distal attachments (a). A color-coded coronal plane section view of the same mechanism is also shown (b), where parts that connect as a rigid body are shaded with the same color. The housing (blue) attaches to the proximal mount that is affixed to the thigh cuff. The pulley (red) rotates with respect to the housing via the main bearing (teal). The output link (green) is affixed to the distal mount that connects to the socket. This link rotates with respect to the pulley via the inner bearing, with the torsional spring connecting the output link to the pulley. The potentiometer measures the relative rotation of the housing and the output link. Hard stops (not shown) are mounted to the pulley assembly and engage with the housing at the limits of joint rotation.

TABLE 1: Design parameters of the powered artificial gastrocnemius.

\begin{tabular}{|c|c|c|}
\hline Parameter & Desired value & Achieved value \\
\hline Joint range of motion (radians) & 1.3 & 2.1 \\
\hline Size & (Minimal) & $50 \mathrm{~mm}$ radius, $53 \mathrm{~mm}$ wide \\
\hline Weight (kg) & (Minimal) & $1.4-1.9$ \\
\hline Center-of-rotation range $(\mathrm{mm})$ & 20 & 20 \\
\hline Maximum output torque (Nm) & 60 & 90 \\
\hline Torque sensing resolution $(\mathrm{Nm})$ & 0.01 & 0.01 \\
\hline
\end{tabular}

2.4. Custom Knee Orthosis and Robotic Ankle-Foot Prosthesis. A custom knee orthosis was built for each amputee participant to use the AG (Figure 4). Each amputee participant was brought to a certified prosthetist to get a cast made of their residual limb while their normal prosthetic socket was being worn. The prosthetist then created carbon fiber orthoses that were form-fitted for each individual. Consequently, an orthosis could be strapped to the affected-side leg while the prosthetic socket was being worn. The orthoses each comprised a carbon fiber thigh cuff and a carbon fiber shank cuff that fitted over the existing prosthetic socket. These cuffs were joined by a pair of polycentric knee orthosis hinges.

A set of bearings was designed to transmit only tangential and sagittal plane forces from the output link of the robotic joint to the shank cuff of the custom orthosis (Figure 4(c)). This bearing set consisted of (1) a linear bearing to allow for distal-proximal freedom between the robotic joint and orthosis, (2) a ball joint to provide 3 -axis rotational freedom, and (3) A linear brass sleeve bearing on a $6 \mathrm{~mm}$ steel shaft to allow medial-lateral freedom.
The orthosis was designed to accommodate a robotic ankle-foot prosthesis (PAFP) (EmPower, BionX Medical Technologies, Inc., Bedford, MA) attached to a given amputee's socket with a standard prosthetic pylon (Figure 4). This prosthesis was a commercial product capable of large amounts of positive net work during walking.

\section{System Dynamics and Torque Control Design}

Despite the large offboard power source, the AG still required system dynamics analyses and control strategies to compensate for parasitic effects like cable friction and other dynamics between the motor and output joint. The goal was to make the system behave as though these dynamics did not exist, so that, from a high-level control standpoint, the AG could be considered close to an ideal torque source. In this section, we describe the characterization of the AG system dynamics. We also describe the development of a low-level torque controller 


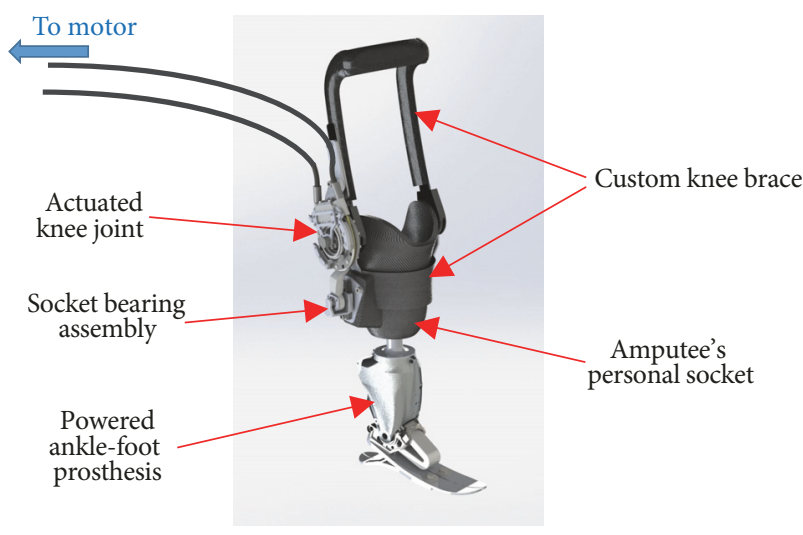

(a)

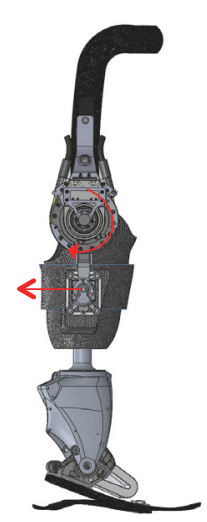

(b)

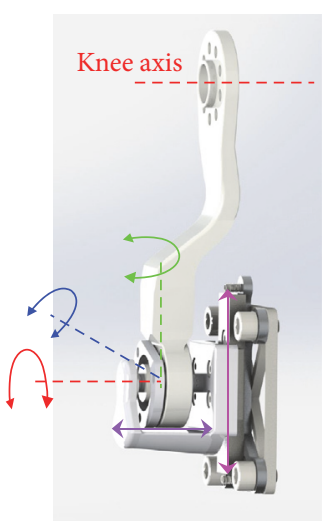

(c)

FIGURE 4: Knee orthosis and socket attachment. Rendering of the powered AG knee orthosis is shown with the robotic knee joint and powered ankle-foot prosthesis (a). A side-view of the same is shown, with arrows indicating the intended applied torque from the knee actuator and the resulting tangential force on the socket from the actuator (b). Also shown is the bearing assembly with resulting degrees of freedom (c) that is mounted on the socket attachment of the orthosis. This assembly transmits the tangential force from the actuator while allowing for motion from the polycentric knee joints as well as misalignment in the orthosis.

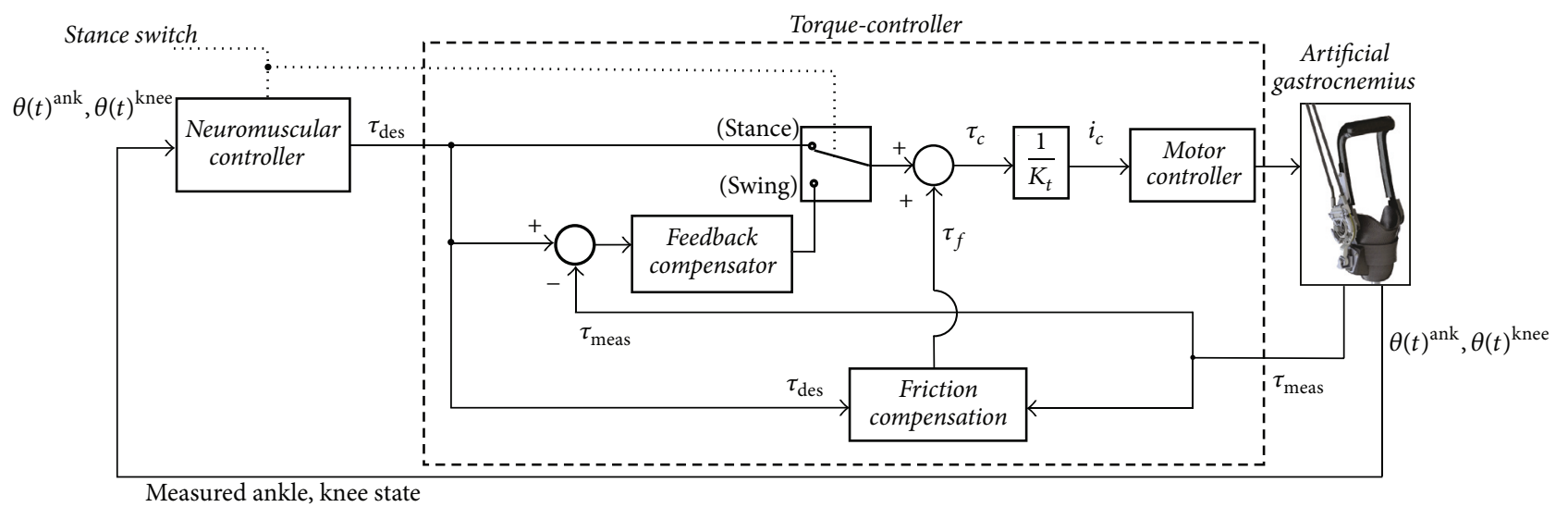

FIgURE 5: Torque control schematic. The control system for the AG is composed of an inner torque-controller loop that enforces desired torque at the joint and an outer loop involving a neuromuscular model as a controller that receives joint kinematics as input. The controller switches between open-loop control (stance) and closed-loop torque control (swing) as appropriate given the gait cycle phase. Motor torque control is performed at the lowest level by controlling motor current, scaled by the torque constant, $K_{t}$.

in terms of torque bandwidth and also performance during walking.

3.1. Open-Loop System Characterization. System identification tests for the AG were performed using a knee orthosis built for nonamputee participants. The orthosis was worn by a healthy adult male participant so to include the full dynamics of the human-machine system. The participant stood still, keeping his knee bent slightly, but resisting motion from the device. A swept-sine torque signal of varying amplitudes from 2 to $10 \mathrm{Nm}$ was commanded to the motor, with frequencies ranging from 0.1 to $200 \mathrm{rad} / \mathrm{s}$ in increments of $10 \mathrm{rad} / \mathrm{s}$. Additional data were collected with the flexible conduit section in the cable conduit linkage bent approximately 15 degrees, so as to capture the range of friction in this section. The magnitude of the command signal was adjusted for each frequency to maximize torque while preventing excessive discomfort to the wearer. Joint moment data were collected from the AG strain gauge. When attached to the human leg as in the system identification experiments, the robotic joint had a $-3 \mathrm{~dB}$ open-loop torque bandwidth of $100 \mathrm{rad} / \mathrm{s}$.

3.2. Artificial Gastrocnemius Torque Control Scheme. The AG was a torque-controlled system. A high-level neuromuscular controller (NMC) took as input affected-side ankle angle $\theta(t)^{\text {ank }}$ and knee angle $\theta(t)^{\text {knee }}$ as functions of time and produced a desired knee joint torque, $\tau_{\text {des }}$. A low-level torque controller enforced this desired torque at the physical joint. For the purposes of this torque control, the gait cycle was divided into two phases: stance and swing (Figure 5). Stance phase began at heel-contact and ended at ToeOff. Swing phase began at ToeOff and ended at subsequent heel-contact. A footswitch (model: FSW, B\&L Engineering, Santa Ana, CA), worn as an insole in the shoe on the leg wearing the knee orthosis, was used to detect stance and swing phases. 
TABLE 2: Amputee matching to nonamputee participants.

\begin{tabular}{|c|c|c|c|c|c|}
\hline Amputee number & Nonamputee match & Amputee height $(\mathrm{cm})$ & Matched height $(\mathrm{cm})$ & Amputee weight $(\mathrm{kg})$ & Matched weight $(\mathrm{kg})$ \\
\hline S1 & NA2 & 180 & 180 & 92 & 90 \\
\hline S2 & NA1 & 175 & 175 & 74 & 73 \\
\hline S3 & NA3 & 193 & 188 & 89 & 95 \\
\hline S4 & NA3 & 188 & 188 & 98 & 95 \\
\hline S5 & NA4 & 175 & 180 & 110 & 103 \\
\hline S6 & NA4 & 180 & 180 & 104 & 103 \\
\hline
\end{tabular}

3.2.1. Stance Phase Torque Control. An open-loop control methodology was used for the stance phase of the gait cycle, wherein the control input was $\tau_{\text {des }}$, and the output was motor torque, $\tau_{c}$ (Figure 5). A feed-forward friction compensation term, $\tau_{f}$ with iterative learning, was used to reduce the torque errors between the desired torque, $\tau_{\text {des }}$ and the measured torque, $\tau_{\text {meas }}$, resulting from friction in the cable drive. This friction compensation was implemented as a torque profile as a function of time within a gait cycle. A running counter was used to estimate the elapsed fraction of the current gait cycle by comparing the time spent in the current gait cycle to the total time of the previous gait cycle: $\mathrm{gc}=$ counter/lastsamples where gc is the estimated elapsed fraction of the current gait cycle, counter is the number of control cycles elapsed in the current gait cycle, and lastsamples is the total number of control cycles of the previous gait cycle.

The function was updated at each gait step by smoothly incorporating the observed torque error in two steps:

$$
\begin{aligned}
\tau_{\text {learn }}(\mathrm{gc}, n+1) & =\tau_{\mathrm{err}}(\mathrm{gc}, n) \cdot \alpha+\tau_{f}(\mathrm{gc}, n) \cdot(1-\alpha), \\
\tau_{f}(\mathrm{gc}, n+1) & =\tau_{\text {learn }}(\mathrm{gc}, n),
\end{aligned}
$$

where gc is the elapsed fraction of the gait cycle, $n$ is the gait cycle number, and $\alpha$ is the learning rate. Once a new learned profile $\tau_{\text {learn }}$ was computed for an entire gait cycle (1), it was subsequently used in the feed-forward term $\tau_{f}$ on the subsequent gait cycle (2). Thus, the error recorded during a gait cycle would be incorporated in the controller two gait cycles later. A value of $\alpha$ of 0.5 was found to provide a reasonable learning rate, for which the learned friction compensation profile was updated such that a new learned behavior was fully incorporated in approximately 10 seconds. Similar iterative learning methods have been successful in wearable robotics in the presence of cable frictional losses [15].

3.2.2. Swing Phase Torque Control. To reduce resistance during swing phase, a feedback torque controller was used along with the iterative friction compensation. This controller took the form

$$
\tau_{\text {comm }}=K_{i} \int\left(\tau_{\text {des }}-\tau_{\text {meas }}\right) d t
$$

where $\tau_{\text {comm }}$ is the motor torque command from the feedback controller. Standard loop-shaping techniques were performed in MATLAB using the open-loop data to determine this control law and the parameter $K_{i}$. In order to provide a fast response during state changes, this torque controller was run throughout the gait cycle, but its output was applied to the robotic joint only during the swing phase.

3.2.3. Closed-Loop System Characterization. System characterization tests were performed for the swing phase feedback torque controller (without the friction compensation), with a swept-sine desired joint torque as input and measured strain gauge joint torque as output, in a similar manner as the open-loop system characterization tests. The closed-loop bandwidth $(-3 \mathrm{~dB})$ and the phase margin were estimated from the data using a fast Fourier transforms of both inputs and outputs. When applying the swing phase closed-loop torque controller, the closed-loop system bandwidth was $14 \mathrm{~Hz}(87 \mathrm{rad} / \mathrm{s})$, with a gain margin of 1.6 and a phase margin of 69 degrees.

3.3. Torque Control Performance during Walking. In addition to the transparency requirement, it was necessary to ensure that the nonzero desired torque values could be tracked by the AG within a reasonable range. The root mean squared (RMS) stance phase torque error, between the desired torque from the NMC and the measured joint torque from the AG strain gauge, was $1.45 \pm 0.72 \mathrm{Nm}$ ( 6 percent of the average maximum torque of $23 \mathrm{Nm}$ ) on average across the amputee participants listed in Table 2. A representative plot of this performance is shown in Figure 6.

3.4. Torque Control Metabolic Validation. Clinical metabolic tests were performed to test the transparency of the robotic knee joint; it was required that the described torque control techniques could provide sufficiently low resistance to wearers as not to adversely affect their metabolism. Success was defined as a nonsignificant difference in metabolism between two conditions during treadmill walking: (1) torquecontrolled AG knee orthosis worn on one leg with zero torque commanded and (2) AG knee orthosis worn, acting as a free-swinging joint (with the cable drive disconnected). For the purposes of this metabolic validation trials with nonamputees, a custom-built, carbon fiber orthosis, molded by a prosthetist, was used to mount the robotic joint on the leg of nonamputee participants.

Six healthy participants (age $=27 \pm 5.7$ years, body mass $=71 \pm 10 \mathrm{~kg}$, and height $=181 \pm 4 \mathrm{~cm}$ ) were asked to walk on a treadmill while metabolic data were collected. The clinical evaluation was conducted at MIT (Cambridge, MA) and was approved by MIT's Committee on the Use of Humans 


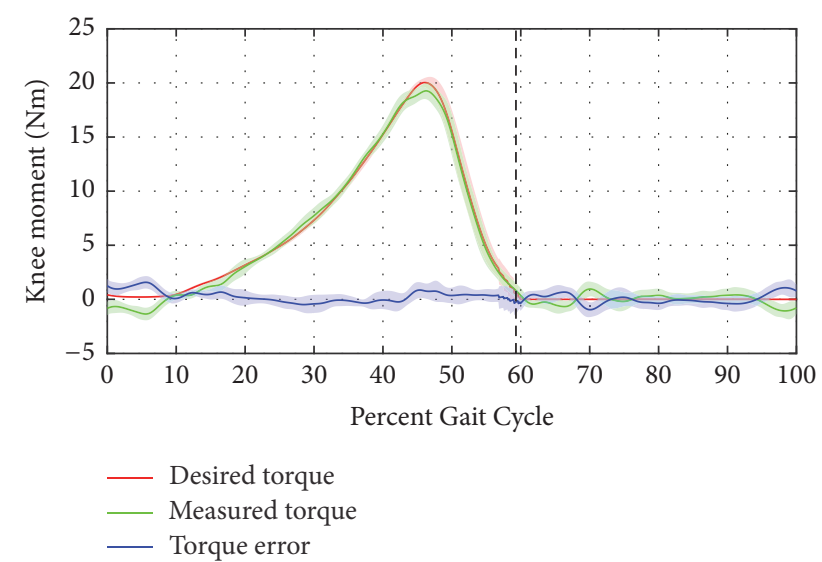

Figure 6: Representative closed-loop system response during walking trials. Data were collected with an amputee participant. The torque error (blue) represents the difference in joint moment between the desired AG device torque (red) and the measured strain-gauge device torque (green). The vertical dashed line indicates ToeOff.

as Experimental Subjects (COUHES). Each participant provided written, informed consent which was obtained before data collection was initiated. Each participant was asked to walk at a typical walking speed of $1.25 \mathrm{~m} / \mathrm{s}$ under three conditions, in random order: (1) walking normally without the AG, (2) wearing the orthosis with the cable drive disconnected, and (3) wearing the orthosis with torque controller set to zero torque. For all conditions, participants wore a portable oxygen consumption mask attached to a standard opencircuit gas analysis system (model $\mathrm{K}_{4} \mathrm{~B}^{2}$, COSMED, Rome, Italy). This system estimated metabolic energy consumption based on measurements of oxygen inspired and expired carbon dioxide.

Metabolic cost for each walking speed was computed by taking average oxygen and carbon dioxide data over a two-minute window at the end of each six-minute trial. The metabolic power was computed using the equation

$$
P=K_{\mathrm{O}_{2}} \dot{V}_{\mathrm{O}_{2}}+K_{\mathrm{CO}_{2}} \dot{V}_{\mathrm{CO}_{2}}
$$

where $P$ is the metabolic power in Watts, $\dot{V}_{\mathrm{O}_{2}}$ is the volume flow rate of oxygen inhaled, $\dot{V}_{\mathrm{CO}_{2}}$ is the volume flow rate of carbon dioxide exhaled, and $\mathrm{K}_{\mathrm{O}_{2}}$ and $\mathrm{K}_{\mathrm{CO}_{2}}$ are constants with values from literature [16], given as $K_{\mathrm{O}_{2}}=16,580 \mathrm{~W} / \mathrm{L}$ and $K_{\mathrm{CO}_{2}}=4,510 \mathrm{~W} / \mathrm{L}$. It was confirmed that the average ratio of $\dot{V}_{\mathrm{CO}_{2}} / \dot{V}_{\mathrm{O}_{2}}$ was less than 1.1 for all trials.

The metabolic consumption of the participants walking with the zero-torque control condition $(3.40 \pm 0.40 \mathrm{~W} / \mathrm{kg})$ was not significantly different from that of the disconnected condition $(3.55 \pm 0.45 \mathrm{~W} / \mathrm{kg}), p=0.18$, although significantly lower metabolism was found for the normal-walking condition without the AG $(2.60 \pm 0.44 \mathrm{~W} / \mathrm{kg}), p<0.001$, as compared to the zero-torque condition.

The results of these system dynamics and metabolic tests show two important performance characteristics. First, the AG can accurately provide high levels of torque to the wearer; the average observed RMS error of $1.45 \mathrm{Nm}$ is well within biological variation; in comparison, the average step-to-step standard deviation in peak knee flexion moment during midstance phase for the nonamputee participants is $4.8 \mathrm{Nm}$. Second, the AG can behave sufficiently close to a free-hinging joint so that the metabolism of the wearer reflects the use of the latter. These two performance characteristics allow the AG to be treated as a near-ideal torque source at both low and high knee moment values. Hence, a higher-level control strategy, such as a neuromuscular controller, may be used without needing to reconsider the system dynamics of the hardware.

\section{Neuromuscular Controller}

A neuromuscular controller (NMC) was used to provide torque commands to the AG so to emulate the behavior of the biological gastrocnemius, similar to earlier work in robotic prosthesis control $[17,18]$. The NMC was based on a musculoskeletal model of a human gastrocnemius. This model took joint kinematics and neural stimulation signals as input and produced joint moments as output. For control purposes, a simulated spinal reflex produced the neural stimulation signals by feeding back the internal state of the musculoskeletal model. Together, the musculoskeletal model and neural reflex took only joint kinematics as input and produced joint moments as output and formed the NMC for controlling the AG.

4.1. Musculoskeletal Model. Nonamputee kinematic, kinetic, and muscle activation data (Appendices A and B) were used to inform the parameter selection of a musculoskeletal model of the human legs. This model comprised a hill-type muscle model for nine effective muscles and attachment geometry of the human leg as described in [19], including optimizer settings and parameter bounds.

The free parameters of the musculoskeletal model were chosen via optimization. The goal was to have the output moments from the optimization closely resemble those from the biological data while keeping simulated metabolic cost close to the measured values. The optimization scheme in [19] was used. For each amputee participant, a corresponding nonamputee was chosen whose height and weight most closely matched that of the given amputee (Table 2). The parameter values of the musculoskeletal model were then optimized based on the walking data at the preferred walking speed for the given nonamputee participant. This preferred speed was that which had the lowest metabolic energy cost per unit distance. This method produced a model that was individually tuned for each amputee.

Several variations of these cost functions have been utilized for determining the parameters of similar models $[19,20]$. In the current work, two methods of evaluating the optimizations were utilized (Appendix C). For both methods, the cost included both kinetic and metabolic similarities of the model to the recorded human walking data. The metabolic cost was represented as cost of transport (COT), which was the metabolic energy cost per unit distance traveled. Upper and lower bounds for the model parameters were 


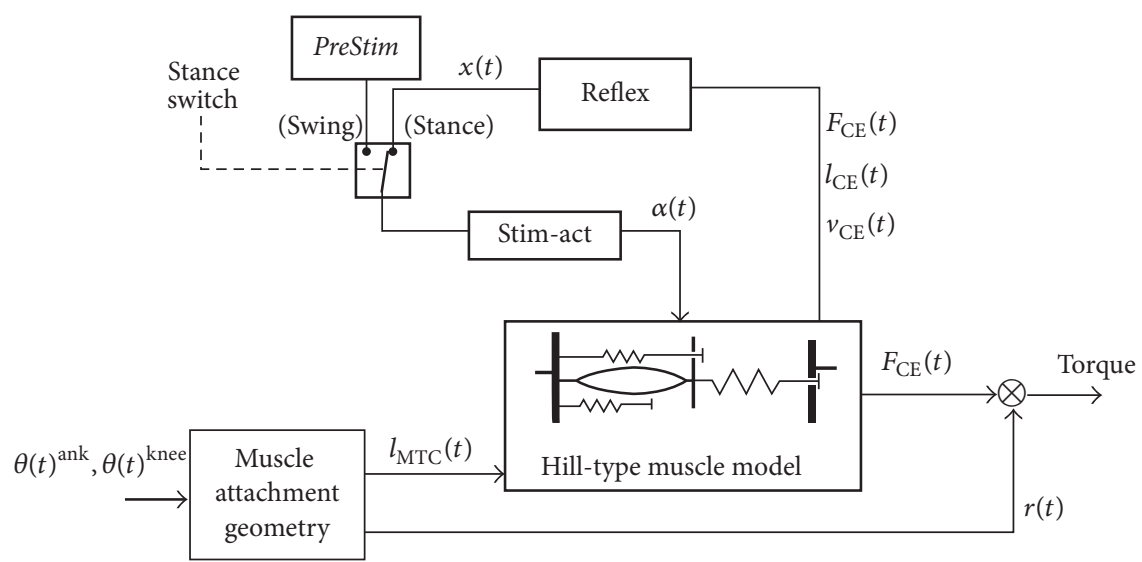

FIGURE 7: Neuromuscular controller including spinal reflex loop. The input to the neuromuscular controller was the kinematics of the ankle and knee joints $\theta(t)^{\text {ank }}$ and $\theta(t)^{\text {knee }}$. The internal reflex loop fed back muscle force, length, and velocity, and produced an internal activation signal to drive the simulated hill-type muscle model. Lookup tables were used to convert from joint kinematics to muscle-tendon length $l_{\mathrm{MTC}}(t)$ and moment arm $r(t)$.

taken from [19]. Optimization settings were also identical to those from [19], except that the present study limited the number of generations to 50, as convergence was observed. See Appendix D for details on the optimization results.

4.2. Spinal Reflex Model. For the purposes of device control, the muscle model required a neural driving signal $x(t)$ to produce the activation $\alpha(t)$. Force, $F_{\mathrm{CE}}(t)$, length, $l_{\mathrm{CE}}(t)$, and velocity, $v_{\mathrm{CE}}(t)$, feedback was used to model the spinal reflex of the gastrocnemius muscle, in the form described in [18] (Figure 7), with a lower bound of the reflex set to PreStim $=0.001$. The stimulation-activation dynamics described in $[21,22]$ were used to obtain $\alpha(t)$ from the spinal reflex signal, $x(t)$, in the same manner as [19]. Additional details can be found in Appendix E.

The reflex gains and threshold values were optimized for the gastrocnemius muscle by simulating the effects of the reflex loop with the musculoskeletal model in the same manner as a previous study in ankle-foot prosthesis control [18]. These parameters were optimized to minimize the mean squared error between the reflex-based activation signals and the muscle EMG patterns from EMG data, given kinematic data. The optimization was performed using a genetic algorithm followed by a gradient descent method. For each data set corresponding to a nonamputee participant, the gastrocnemius morphological parameters of the musculoskeletal model were taken from the results of the corresponding morphological optimization.

4.3. Neuromuscular Controller Implementation. For the purpose of prosthesis control, the musculoskeletal model took as input walking state information and produced desired torque commands to the robotic joint during the stance phase. The ankle joint angle was read from an electrogoniometer (Delsys, Boston, MA) which measured the affected-side ankle angle, and the affected-side knee joint angle was read from the AG onboard joint angle sensor. The NMC was disabled during the swing phase by setting the neural stimulation to zero using the stance phase switch and ignoring any residual commanded torque. The muscle-tendon length and moment arm geometry were encoded in lookup tables to allow realtime computation of these values from joint kinematics. For additional tuning, the force gain of the reflex controller was made adjustable in real-time. This gain was chosen for adjustment since the controller's output power in simulation, based on nonamputee data, was found to be the most sensitive to changes in this parameter. The NMC was implemented in MATLAB Simulink and compiled to a $\mathrm{C}$ executable that communicated at $1 \mathrm{kHz}$ to the master controller that, in turn, controlled the motor.

For each amputee participant, a corresponding model was used, which was based on a height-weight matched nonamputee participant's walking data (Table 2). The COT values for all nonamputee participants across walking speed are shown in Table 3 . The participants all had minimal COT at either 1.25 or $1.5 \mathrm{~m} / \mathrm{s}$.

With this neuromuscular controller, the AG emulated the behavior of a biological gastrocnemius muscle by taking only the sensor-based ankle and knee joint kinematics as input. Thus, the AG was a self-contained unit that acted as an externally worn gastrocnemius muscle with neural and musculoskeletal dynamics encoded in its control.

\section{Clinical Trials}

In the final clinical trials, six participants with transtibial amputation walked on an instrumental treadmill while wearing a PAFP-AG combination while biomechanical and metabolic data were collected (Figure 2(a)).

5.1. Experimental Setup. The walking speed for a given amputee was set as the preferred speed determined from the matched nonamputee walking data (Table 3 ). This speed was set for all walking conditions. The clinical evaluation was conducted at MIT (Cambridge, MA) and was approved by MIT's Committee on the Use of Humans as Experimental Subjects (COUHES). Each participant provided written, informed consent before data collection was initiated. 
TABLE 3: Metabolic cost of transport for nonamputee participants.

\begin{tabular}{lccccccc}
\hline Participant & $0.75 \mathrm{~m} / \mathrm{s}$ & $1.0 \mathrm{~m} / \mathrm{s}$ & $1.25 \mathrm{~m} / \mathrm{s}$ & $1.5 \mathrm{~m} / \mathrm{s}$ & $1.75 \mathrm{~m} / \mathrm{s}$ & $2.0 \mathrm{~m} / \mathrm{s}$ & Optimal speed $(\mathrm{m} / \mathrm{s})$ \\
\hline NA1 & 0.419 & 0.364 & 0.326 & 0.339 & 0.362 & 0.380 & 1.25 \\
NA2 & 0.462 & 0.386 & 0.371 & 0.375 & 0.404 & 0.467 & 1.25 \\
NA3 & 0.481 & 0.389 & 0.347 & 0.341 & 0.368 & 0.400 & 1.5 \\
NA4 & 0.395 & 0.339 & 0.305 & 0.315 & 0.348 & 0.408 & 1.25 \\
\hline
\end{tabular}

Each amputee was first asked to don only the PAFP, and the electrogoniometer was installed on the PAFP for subsequent use with the AG. The participant was then instructed to walk on the treadmill while the prosthesis power was adjusted using the commercial tuning app so to achieve average prosthesis power within two standard deviations from the average biological ankle power at the given walking speed [23]. Prosthesis tuning was subsequently adjusted slightly during subsequent trials as needed in an attempt to maintain prosthesis power within these limits.

Next the amputee donned the AG orthosis along with the PAFP. A short time period was given to ensure that both devices were functioning properly. Then the amputee was asked to walk on an instrumented treadmill at the given walking speed with the same data collection methods used for developing the NMC, including the instrumented treadmill, and reflective markers. Each trial consisted of 90 seconds of data collection.

In addition to using the optimized parameters of the NMC, additional conditions were tested wherein the force feedback reflex gain of the NMC was adjusted manually. This adjustment allowed some level of controller tuning based on the actual participant feedback. While a participant walked on the treadmill with the AG and PAFP, this gain was incrementally increased from its optimized, Default value (the Default gain setting) until either further increases in gain were said to be undesirable by the participant or the average power from the device was no longer increasing with higher controller gain (the Max gain setting). The gain was then decreased until the average power coming from the device was in the range of the biological gastrocnemius, of 3-5 Joules (the BioGain setting) $[12,13]$. If the Default setting provided less than or equal to 3-5 Joules of net mechanical work per step, an additional gain was set to mid-way between the Default gain and the Max gain. This additional gain setting was called Half.

Once the aforementioned conditions were recorded with the motion capture system, metabolic data were collected using the same gas analysis system as the other data collection sessions (see Section 3.4). The participants were first asked to perform one standing trial to measure standing metabolism. Next, the walking conditions described above were repeated, in random order. Each metabolic trial lasted 6 minutes.

5.2. Data Processing. The same data processing methods as in the NMC development (see Appendix B) were used to obtain gait events, sagittal plane joint moments and angles, and metabolic cost from the recorded data, except that a point-mass of $2 \mathrm{~kg}$ was added at the affected-side knee joint of the SIMM model to represent the dynamical effects of the robotic joint and conduit mass. Also affected-side biological knee moment contribution was computed by subtracting the measured AG knee moment from the total knee moment estimated from the inverse dynamics.

The walking metabolism was compared for each amputee participant across trials, where each trial involved a different value for the force feedback reflex gain of the NMC. The optimal gain was found as the one that resulted in the lowest metabolic power for a given amputee. The walking condition (called the OPT condition) corresponding to this optimal gain was then compared to the walking condition where the AG was controlled to behave as a free-joint (called the ZERO condition).

Joint powers were computed as the product of joint velocity from joint kinematics and joint moments derived from the motion capture data, where positive power was defined as that produced by the joint on the environment. Where available, mechanical power from the powered prosthesis was taken from PAFP telemetry data, computed using the prosthesis onboard torque and angle sensors. For two subjects (S2 and S3), these data were not available, so PAFP mechanical power was computed in the same manner as the other joints, using SIMM-derived ankle angular velocities and moments from the motion capture data. AG applied mechanical power was computed from motion capture-based knee joint angular velocity and internal AG torque from strain gauge data.

Positive and negative joint work contributions were defined as the time integral of the positive and negative components of joint power, respectively, integrated over the late stance knee flexion phase (from mid-stance maximum knee extension to ToeOff), and divided by the time in that phase. Flexion and extension moment impulse values were computed in the same way, but using joint moments instead of powers.

Theoretical reductions in metabolic power were computed by assuming the average mechanical power provided by the combined AG knee actuator and ankle prosthesis in the OPT condition, as compared to the ZERO condition (with zero net work assumed for the AG knee in the ZERO condition), could perfectly replace muscle fiber mechanical power. With the efficiency of skeletal muscle assumed to be $25 \%$ [24], the reduction in muscle metabolic power was computed as 4 times the observed mechanical power provided by the AG knee actuator and ankle-foot prosthesis in the OPT condition. The resulting change in metabolic power was then compared to the measured metabolic power from the ZERO condition to determine theoretical percent reduction.

Statistics were run on metabolic powers and also bilateral ankle, knee, and hip joint features (flexion and extension 
TABLE 4: Walking conditions for minimal metabolic power (OPT condition). For all but the last row, numbers represent the settings and results during the OPT condition. In the last row, positive values indicate that the given robotic joint produced greater net work in the OPT condition than in the ZERO condition.

\begin{tabular}{|c|c|c|c|c|c|c|}
\hline Participant & S1 & S2 & S3 & S4 & S5 & S6 \\
\hline Condition name & Bio & $\operatorname{Max}$ & Default & Default & Default & Half \\
\hline Force gain scaling & 0.1 & 8 & 1 & 1 & 1 & 1.5 \\
\hline Force gain $(1 / \mathrm{N})$ & $3.69 e-5$ & $4.99 e-3$ & $3.99 e-4$ & $6.19 e-4$ & $5.11 e-4$ & $7.66 e-4$ \\
\hline AG mean power $(\mathrm{W} / \mathrm{kg})$ & 0.054 & 0.118 & 0.028 & 0.027 & 0.105 & 0.031 \\
\hline Change in AG mean power from ZERO $(\mathrm{W} / \mathrm{kg})$ & -0.001 & +0.020 & +0.056 & +0.077 & +0.011 & +0.009 \\
\hline
\end{tabular}

TABLE 5: Metabolic cost of transport using the artificial gastrocnemius. Values given represent the net metabolic power, above that of basal metabolic rate. Positive values for percent change indicate that the participant required less metabolic power with the neuromuscular controller, compared to the control condition of zero-torque at the knee. Theoretical metabolic reduction, based on the change in knee orthosis and ankle-foot prosthesis applied average power from the ZERO condition to the OPT condition, and assuming $25 \%$ muscle efficiency, is also shown for comparison.

\begin{tabular}{|c|c|c|c|c|c|c|}
\hline & S1 & S2 & S3 & S4 & S5 & S6 \\
\hline ZERO Condition Net Metabolic Power (W/kg) & 2.95 & 3.51 & 3.60 & 5.06 & 3.34 & 3.52 \\
\hline OPT Condition Net Metabolic Power (W/kg) & 3.13 & 3.08 & 3.87 & 4.95 & 2.97 & 3.38 \\
\hline Percent change in metabolic power from ZERO condition to OPT condition & 5.8 & -12.1 & 7.4 & -2.3 & -11.1 & -4.0 \\
\hline Theoretical percent change in metabolic power & -7.2 & -15.7 & -9.3 & -8.3 & -14.1 & -4.6 \\
\hline
\end{tabular}

moment impulses and positive and negative joint work contributions) by performing a two-sided, paired $t$-test between these features during the OPT condition and ZERO condition. Statistically significant differences were defined as those with $p$ values less than or equal to 0.05 .

\subsection{Clinical Results}

5.3.1. AG Overall Device Performance. In the final amputee trials with the AG-PAFP combination, the metabolically optimal (OPT) conditions were found for each participant, based on the adjusted parameter of the force-reflex gain. The gain settings and average output power from the AG joints are summarized in Table 4.

5.3.2. Metabolic Power. Walking metabolism is shown in Table 5 for the walking conditions in which the metabolic cost was found to be lowest, compared to the theoretically calculated values using device average power. Large percentage reductions in metabolism were found in two of the six participants. Two participants showed small metabolic reductions, and the two remaining participants had metabolism values higher with the AG intervention, compared to the zerotorque condition. Within the participants with a metabolic reduction in the OPT condition, these metabolic reductions were well correlated with the theoretical values based on device mechanical power $(r=0.9)$, as shown in Table 5 . However, Subject 1 and Subject 3 displayed increases in metabolism in the OPT condition, as compared to the ZERO condition, which was not predicted with the theoretical calculations. Overall, the metabolic power between the ZERO and OPT conditions was not found to be statistically significant $(p=0.43)$.

5.3.3. Joint Kinetics. Comparing the ZERO and OPT conditions, statistically significant differences were found in the

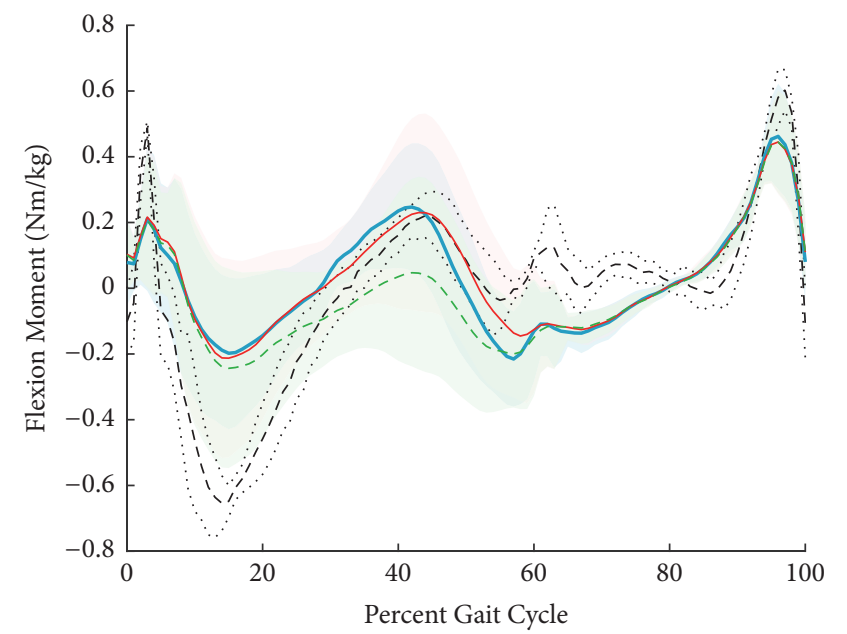

Figure 8: Components of affected-side knee moment of amputee participants with the powered AG, averaged across participants. The net value, which is the sum of biological knee and AG values, is shown for two walking conditions: the ZERO condition (thick solid blue line) and the OPT condition (thin solid red line). The contribution from the biological knee joint is also shown for the OPT condition (dashed thin green line). Nonamputee data, averaged across participants, are shown for reference, with the mean (dashed black line) \pm one standard deviation (dotted black lines).

biological knee flexion moment impulse and biological hip positive work of the affected leg during the late-stance knee flexion phase of gait. The knee flexion moment impulse was reduced by an average of $0.0223 \mathrm{Nm} * \mathrm{~s} / \mathrm{kg}(p=0.03)$, from the ZERO condition $(0.0413 \pm 0.0262 \mathrm{Nm} * \mathrm{~s} / \mathrm{kg})$ to the OPT condition $(0.0190 \pm 0.0252 \mathrm{Nm} * \mathrm{~s} / \mathrm{kg})$. Figure 8 illustrates the $\mathrm{AG}$ and biological knee moment contributions over the gait cycle. The affected-side hip showed a decrease in positive 
work of $0.0739 \mathrm{~J} / \mathrm{kg}(p=0.004)$ from the ZERO condition $(0.2621 \pm 0.0848 \mathrm{~J} / \mathrm{kg})$ to the OPT condition $(0.1882 \pm$ $0.0734 \mathrm{~J} / \mathrm{kg}$ ). All other tested kinetic features did not indicate significant differences between conditions.

5.4. Discussion. Although a statistically significant metabolic reduction across participants was not found in this study, the large percentage reductions in metabolism for some participants, as in Table 5, indicate that it is likely that a metabolic advantage may be achieved with a powered artificial gastrocnemius, provided appropriate conditions are satisfied. It is yet unclear what exact conditions must be met to provide metabolic reductions for all participants. However, within the participants with a metabolic reduction in the OPT condition, these reductions were fairly well correlated with the theoretical values based on device mechanical power $(r=0.9)$, as shown in Table 5. Therefore, it is possible that higher levels of mechanical power from the AG would increase the chance of producing a statistically significant metabolic reduction across participants.

The kinetic changes, as also illustrated in Figure 8, support the hypothesis that the AG causes a reduction in affected biological knee flexion moment impulse and hip positive net work, but not knee positive work or hip moment impulse. Considering the short trial times, this result indicates that participants were capable of rapid kinetic responses to the interventions, on the order of several minutes or less. The knee moment impulse reduction may stem from an attempt by the participants to prevent the total knee moment from changing in the presence of the external disturbance. This result is consistent with other studies, as humans have been observed to maintain invariant joint moments at both the ankle [25] and hip joints [26] when an external torque from an exoskeleton is applied. This finding has a potential consequence: if the applied joint moment from the AG exceeded the affected knee moment from the ZERO condition, the participant might attempt to maintain total joint kinetics by providing an active extension moment with their quadriceps, so to prevent the total knee flexion moment from increasing. This behavior may put an upper-limit on the flexion torque that can be provided by the AG without incurring a cost from the quadriceps in the form of active knee extension efforts.

5.4.1. Potential Mechanisms for Metabolic Reductions. First, it is possible that this reduction in knee moment itself may play a role in the metabolic reduction in some of the participants, as the production of muscle force exacts a metabolic cost [27]. However, the generation of isometric muscle force alone is comparatively less costly compared to the generation of mechanical work. Therefore, other factors are likely at play.

The similarity of the observed metabolic reductions to the theoretical values (Table 5) suggests that much of the power provided by the AG goes to replacing muscle fiber positive work, as modeled in the theoretical cost values. The reduction in affected-side hip positive work with AG assistance indicates that energy may be transferred from the AG up to the hip from the knee. These hip work reductions seen in late-stance knee flexion coincide with the initiation of hip flexion during preswing, indicating that the AG probably helps to initiate leg swing. This result is consistent with what is known about the normal function of the human gastrocnemius muscle during walking $[28,29]$.

\section{Conclusions and Future Work}

To create the first fully powered artificial gastrocnemius for transtibial amputees, we develop a versatile knee orthosis that is capable of kinetic behavior comparable to the biological gastrocnemius. The control strategies provide low errors between desired and measured joint torque across a wide dynamic range. Good torque tracking of zero-torque commands allow for metabolic transparency, while the torque tracking at higher torque levels enables the system to replicate desired behavior. This large dynamic range is attributed to the tethered system that enabled large power capability without the commensurate cost of added actuator mass. Others have made use of this type of platform for this reason [30,31]. Unlike other researchers, however, our use of a cable linkage mitigates transmission losses in the cable drive compared to a pure Bowden cable transmission, since only part of the cable is in contact with the conduit surfaces. This system is well suited for simulating a gastrocnemius muscle at the knee for amputee participants.

The clinical study presented here represents an effort to improve upon earlier biarticular transtibial prostheses by using an active element for the affected knee joint. Such an active device can provide positive net work, unlike previous quasi-passive counterparts $[10,11]$. If this net robotic work replaces positive muscle work, we expect to see large reductions in metabolism with the biarticular conditions, as compared to the monoarticular conditions. Indeed, with the application of sufficient positive work, the results suggest that it may be possible to provide metabolic and kinetic assistance to transtibial amputee walking gaits beyond that provided by a powered ankle-foot prosthesis alone. This study also provides suggestive evidence that metabolic improvements may stem from not only reductions in muscle activity at the assisted joint, but other joints as well, such as the hip. This finding is consistent with other work in lower-extremity exoskeletons [32].

However, this study also highlights the difficulty of reducing metabolic demand in humans, even in the presence of an existing pathology. Even the smallest unwanted perturbations can severely hinder an intervention that would otherwise cause a successful metabolic improvement. Thus, in the development of assistive wearable devices, it is of utmost importance to consider the biomechanical consequences of applying torques and powers to the human body. Future work could utilize a torque control strategy to more systematically vary output power levels of the AG to determine if higher net positive work could further improve metabolic cost of the wearer, as researchers have performed with an ankle exoskeleton [33]. An additional next step would be to use a modified NMC to command both the knee and ankle joints of the powered artificial gastrocnemius with one, unified model. Indeed, such a control strategy would provide better synchronization between the knee and ankle actuators. 
TABLE 6: Nonamputee body parameters.

\begin{tabular}{lcc}
\hline Participant number & Height $(\mathrm{cm})$ & Weight $(\mathrm{kg})$ \\
\hline NA1 & 175 & 73.4 \\
NA2 & 180 & 90.3 \\
NA3 & 188 & 95.4 \\
NA4 & 180 & 103 \\
\hline
\end{tabular}

In the advancement of active transtibial prostheses, we feel a powered artificial gastrocnemius is of critical importance. It is our hope that this study will underscore the potential of this novel mechatronic approach to the biomechanical and physiological gait performance of persons with transtibial leg amputation.

\section{Appendix}

\section{A. Neuromuscular Controller Data Collection}

The parameters of the neuromuscular controller (NMC) were selected in two stages. In the first stage, the musculoskeletal model parameters were optimized for a full leg musculoskeletal model so to best match biological joint moments and metabolism, given biological kinetics and EMG data. In the second stage, the morphological model parameters for the gastrocnemius were fixed at their optimized values, and the reflex parameters were optimized for this muscle.

The gait data of four healthy, nonamputees were used to inform the parameters of the NMC. These nonamputee participants were chosen so to match the amputees as closely as possible (see Table 6). The clinical evaluation was conducted at MIT (Cambridge, MA) and was approved by MIT's Committee on the Use of Humans as Experimental Subjects (COUHES). Each participant provided written, informed consent which was obtained before data collection was initiated. These data were collected in two phases.

In the first phase, kinematic, kinetic, and electromyographic (EMG) data were collected for 90 seconds at each of six speeds $(0.75 \mathrm{~m} / \mathrm{s}, 1.00 \mathrm{~m} / \mathrm{s}, 1.25 \mathrm{~m} / \mathrm{s}, 1.50 \mathrm{~m} / \mathrm{s}, 1.75 \mathrm{~m} / \mathrm{s}$, and $2.00 \mathrm{~m} / \mathrm{s}$ ) in random order. An infrared camera system (12 cameras, model: T40s, Vicon Motion Systems Ltd., Oxford, UK) was used to track the motion of subjects as they walked in the capture volume. Reflective markers were placed at 47 locations on each participant's body, based on the Helen Hayes marker model, and their threedimensional trajectories were recorded at $100 \mathrm{~Hz}$. The marker locations were chosen specifically to track joint motion. The ground reaction forces and contact centers of pressure were measured using an instrumented force plate treadmill (Bertec Corporation, Columbus, $\mathrm{OH}$ ) with a sampling rate of $1 \mathrm{kHz}$. Electromyographic signals were collected at $2 \mathrm{kHz}$ using a wireless surface system (Trigno, Delsys Inc., Natick MA). Twelve muscles (tibialis anterior, soleus, medial gastrocnemius, lateral gastrocnemius, rectus femoris, vastus lateralis, vastus medialis, semimembranosus, biceps femoris long head, adductor magnus, iliacus, and gluteus maximus) on the right leg of each subject were recorded, with symmetry being assumed for the other leg. Prior to the walking trials, the muscle maximum voluntary contraction (MVC) tests were performed for each of the muscles of interest, in which the participants were instructed to push as hard as possible, three times in succession for 3-5 seconds each with the given muscle group, while electromyographic data were collected.

Once the first data collection phase was completed, the markers and electrodes were removed, and the participants were equipped with an open-circuit gas exchange measurement system for estimating metabolic cost (model: K4b2, COSMED, Rome, Italy). The participants were asked to perform one standing trial to measure standing metabolism, and then walking trials were performed on the treadmill for the same six walking speeds. Each walking trial lasted six minutes.

\section{B. Neuromuscular Controller Data Processing}

Fourth-order Butterworth filters were used to filter the marker position and ground reaction force data with $6 \mathrm{~Hz}$ and $25 \mathrm{~Hz}$ cutoff frequencies, respectively. The marker and force data were postprocessed through the inverse dynamics module of SIMM (MusculoGraphics Inc., Evanston, IL), to produce joint moments and angles for ankle, knee, and hip joints, as well as muscle-tendon lengths and moment arms, all as a function of time. Only sagittal plane dynamics were considered.

Gait events were determined using vertical ground reaction force data from the embedded force plates. Approximate event timing was found by determining the times when the force increased beyond a $40 \mathrm{~N}$ threshold. Exact heel strike and ToeOff times where found by progressing backward and forward in time, respectively, until the force value dropped to zero. Data were then cut to gait cycles based on heel strike times and resampled to 101 points. Gait cycles were discarded for the beginning and end of each trial, during the speed transients of the treadmill. Gait cycles in which the stride times were below 0.7 seconds or above 1.3 seconds or in which a foot crossed the midline of the treadmill were also discarded. Metabolic cost for each walking speed was computed in the same manner as in the nonamputee validation trials (Section 3.4).

Several processing steps were taken to prepare the EMG signal to be processed for the estimation of muscle activation. EMG signals were first processed internally by the EMG system by applying a 4 th-order bandpass filter with a pass band from $20 \mathrm{~Hz}$ to $450 \mathrm{~Hz}$. A $60-\mathrm{Hz}$ notch filter was also applied to remove mains hum. Any DC offset was removed, and the remaining signal was saturated at 5 standard deviations from zero. The signal was then normalized to the new maximum value of 5 standard deviations and rectified.

The processed EMG data were further processed with the method described in [19] to achieve estimates of muscle activation from the measured EMG signals. The activation was initially scaled by those resulting from the MVC trials, but the resulting scaled EMG signals were unreasonably scaled when compared to literature values. Therefore, the final magnitudes of the estimated activation signals were scaled so that the average values of the activation signals 
TABLE 7: Optimized morphological parameters.

\begin{tabular}{lcccccc}
\hline Parameter & S1 & S2 & S3 & S4 & S5 & S6 \\
\hline$F_{\max }$ & 1094 & 1052 & 1094 & 1936 & 3049 & 3049 \\
$l_{\text {sl }}$ & 0.4565 & 0.4115 & 0.4565 & 0.4568 & 0.4217 & 0.4217 \\
$l_{\text {opt }}$ & 0.0504 & 0.0454 & 0.0504 & 0.0504 & 0.0465 & 0.0465 \\
$K_{\text {sh }}$ & 2.58 & 2.78 & 2.58 & 2.722 & 2.84 & 0.0599 \\
$\lambda_{\text {ref }}$ & 0.0593 & 0.0684 & 0.0593 & & 0.0846 \\
\hline
\end{tabular}

matched those from [34] during the range of activation for the literature data.

As was found in a previous study [19], many of the muscles spanning the hip were found to have signals too weak to be reliably measured (rectus femoris, iliacus, biceps femoris short head, hip abductors, adductor longus, and adductor magnus). For these muscles, neural excitation profiles from wire electrode experiments [34] were delayed $40 \mathrm{~ms}$ to simulate the delay between the EMG signals and the development of force [35] and then passed through the same activation dynamics used for the collected electromyographic data. The resulting activation signals were then used as input to the musculoskeletal model along with those derived from the electromyography.

\section{Morphological Optimization Cost Functions}

In the current work, two methods of evaluating the morphological model parameters were utilized. For the first cost function (used for Subjects 2 and 3), the musculoskeletal model parameters were optimized using the method described by [19]. However, using this method, it was found that the measured electromyographic data failed to produce satisfactory values for both kinetic fits and metabolic cost estimates; either kinetic fit $R^{2}$ values were low (less than a cutoff threshold of 0.7) or the metabolic cost estimates of the model were more than one standard deviation higher than the mean of the empirical metabolic cost values (from the nonamputee participants walking at their preferred speeds). As an alternative, the activation profiles derived from wire electrode experiments [34] provided both kinetic and metabolic results closer to that of biology. Therefore, for this optimization method, all muscle activation profiles were derived from the wire electrode literature [34], processed through the same activation dynamics used for the EMG [19]. Selection of the final parameter set was also performed as in [19]. For Subject 2, the HAB muscle group was used for selecting the final parameter set. For Subject 3, the VAS muscle group was used. These muscles were selected based on their dominance in the simulated fractional metabolic cost.

Some biological walking data did not produce acceptable kinetic and metabolic cost values (as described above) with the first optimization method (those for Subjects 1, 5, and 6), or the resulting model produced low average mechanical power when tested in simulation with walking kinematics and EMG as input (Subject 4). For these data sets, a different cost function was used. This metabolic cost estimation function was based on empirical measures of muscle metabolic energy consumption as a function of fiber contractile velocity
TABLE 8: Final cost values for the morphological parameter optimization. The COT values were the simulated metabolic cost of transport values, as computed by the musculoskeletal model, when given the final optimized parameter set and biological nonamputee data as input.

\begin{tabular}{lcccccc}
\hline Cost value & S1 & S2 & S3 & S4 & S5 & S6 \\
\hline$R^{2}$ & 0.71 & 0.76 & 0.80 & 0.85 & 0.80 & 0.80 \\
COT & 0.360 & 0.324 & 0.246 & 0.330 & 0.301 & 0.301 \\
\hline
\end{tabular}

and activation [36, 37]. The simulated metabolic cost was found by integrating muscle power for each muscle in the leg over the gait cycle, and summing up all muscles for the leg, assuming bilateral symmetry. The measured basal energy consumption was added to the resulting value to determine the full-body metabolic energy cost. As with the first cost function, both the simulated metabolic cost and the average kinetic fit were considered for the evaluation of parameter solutions. However, it was found that the kinetic fit was largely invariant in the range of solutions where the simulated COT was near that of the measured values. Therefore, in selecting a single solution, the parameter set with the best kinetic fit was chosen, provided that the simulated COT was within the standard error of the measured COT value. A similar parameter selection method had been used to select optimal parameter sets in previous work [38].

\section{Morphological Parameter Optimization Results}

The optimized morphological parameters for the gastrocnemius muscle are shown in Table 7 . The cost values are shown in Table 8. These parameters correspond to those in the model described in previous work [19].

\section{E. Spinal Reflex Model}

The muscle model required a neural driving signal $x(t)$ to produce the activation $\alpha(t)$. Herein, the neural driving signal can be represented as [18]

$$
\begin{aligned}
x(t)= & G_{F} \cdot\left(F\left(t-\Delta t_{F}\right)-F_{0}\right) \cdot u\left(F-F_{0}\right)+G_{l} \\
& \cdot\left(l\left(t-\Delta t_{l v}\right)-l_{0}\right) \cdot u\left(l-l_{0}\right)+G_{v} \\
& \cdot\left(v\left(t-\Delta t_{l v}\right)-v_{0}\right) \cdot u\left(|v|-v_{0}\right) .
\end{aligned}
$$

Here, $G_{F}, G_{l}$, and $G_{v}$ are the gains; $\Delta t_{F}, \Delta t_{l}$, and $\Delta t_{v}$ are the time delays for the force, muscle length, and muscle velocity 
TABLE 9: Optimized neuromuscular model reflex parameters. Each column represents the settings for an amputee participant, with the corresponding nonamputee data set in parentheses.

\begin{tabular}{lcccccc}
\hline & S1 (NA2) & S2 (NA1) & S3 (NA3) & S4 (NA3) & S5 (NA4) & S6 (NA4) \\
\hline$G_{F}(1 / \mathrm{N})$ & $3.687 e-4$ & $6.238 e-4$ & $3.994 e-4$ & $6.185 e-4$ & $5.11 e-4$ & $5.11 e-4$ \\
$F_{0}(\mathrm{~N})$ & 0.1786 & 0.3935 & 0.3624 & 0.291 & 0.532 & 0.532 \\
$G_{l}(1 / \mathrm{m})$ & 6.88 & 5.22 & 1.3465 & 6.32 & 8.19 & 8.19 \\
$l_{0}(\mathrm{~m})$ & 0.0507 & 0.0490 & 0.0528 & 0.0448 & 0.0382 & 0.0382 \\
$G_{v}(\mathrm{~s} / \mathrm{m})$ & 0.9606 & 1.34 & 2.00 & 0.0193 & 2.00 & 2.00 \\
$v_{0}(\mathrm{~m} / \mathrm{s})$ & 0.1230 & -0.1175 & -0.1168 & -0.0897 & 0.0340 & 0.0340 \\
\hline
\end{tabular}

values, respectively. The function $u(\cdots)$ is a step function, indicating that each reflex only activates for values greater than the corresponding threshold value $\left(F_{0}, l_{0}\right.$, and $v_{0}$ for force, length, and velocity, resp.). The time delays model the neural delay for a signal to traverse the reflex arc from muscle to spinal cord and back to the muscle. All three delays were fixed at $20 \mathrm{~ms}$ [39-41]. The reflex signal $x(t)$ represents the neural stimulation. The optimized parameter values for the reflex loop are shown in Table 9. The stimulation-activation dynamics described in previous work [19] were used to obtain the muscle activation, $\alpha$.

\section{Conflicts of Interest}

The authors declare no conflicts of interest regarding the publication of this article.

\section{Acknowledgments}

This work was supported by NASA [NNX12AM16G] as part of the National Robotics Initiative and departmental funding from the MIT Media Lab. The authors thank Bob Emerson and Jared Markowitz for sharing their valuable expertise.

\section{References}

[1] N. H. Molen, "Energy/speed relation of below-knee amputees walking on a motor-driven treadmill," Internationale Zeitschrift für Angewandte Physiologie Einschließlich Arbeitsphysiologie, vol. 31, no. 3, pp. 173-185, 1973.

[2] R. L. Waters, J. Perry, D. Antonelli, and H. Hislop, "Energy cost of walking of amputees: the influence of level of amputation," The Journal of Bone \& Joint Surgery, vol. 58, no. 1, pp. 42-46, 1976.

[3] G. Colborne, S. Naumann, P. Longmuir, and D. Berbrayer, Am J Phys Med Rehabil, vol. 71, no. 5, pp. 272-278, 1992.

[4] P.-F. Su, S. A. Gard, R. D. Lipschutz, and T. A. Kuiken, "Gait characteristics of persons with bilateral transtibial amputations," Journal of Rehabilitation Research and Development, vol. 44, no. 4, pp. 491-501, 2007.

[5] H. M. Herr and A. M. Grabowski, "Bionic ankle-foot prosthesis normalizes walking gait for persons with leg amputation," Proceedings of the Royal Society B Biological Science, vol. 279, no. 1728, pp. 457-464, 2012.

[6] D. Hill and H. Herr, "Effects of a powered ankle-foot prosthesis on kinetic loading of the contralateral limb: a case series," in Proceedings of the IEEE International Conference on Rehabilitation Robotics (ICORR '13), pp. 1-6, Seattle, Wash, USA, June 2013.
[7] M. R. Williams, A. Grabowski, H. Herr, and S. D’Andrea, "Electromyographic effects of using a powered ankle-foot prosthesis," American Society of Biomechanics, 2012.

[8] E. Isakov, O. Keren, and N. Benjuya, "Trans-tibial amputee gait: Time-distance parameters and EMG activity," Prosthetics and Orthotics International, vol. 24, no. 3, pp. 216-220, 2000.

[9] E. Isakov, H. Burger, J. Krajnik, M. Gregoric, and C. Marincek, "Knee muscle activity during ambulation of trans-tibial amputees," Journal of Rehabilitation Medicine, vol. 33, no. 5, pp. 196-199, 2001.

[10] K. Endo, E. Swart, and H. Herr, "An artificial gastrocnemius for a transtibial prosthesis," in Proceedings of the 31st Annual International Conference of the IEEE Engineering in Medicine and Biology Society: Engineering the Future of Biomedicine, EMBC 2009, pp. 5034-5037, USA, September 2009.

[11] M. F. Eilenberg, K. Endo, and H. Herr, "Biomechanic and Energetic Effects of a Quasi-Passive Artificial Gastrocnemius on Transtibial Amputee Gait," Journal of Robotics, In press.

[12] R. J. Zmitrewicz, R. R. Neptune, and K. Sasaki, "Mechanical energetic contributions from individual muscles and elastic prosthetic feet during symmetric unilateral transtibial amputee walking: A theoretical study," Journal of Biomechanics, vol. 40, no. 8, pp. 1824-1831, 2007.

[13] R. R. Neptune, K. Sasaki, and S. A. Kautz, "The effect of walking speed on muscle function and mechanical energetics," Gait \& Posture, vol. 28, no. 1, pp. 135-143, 2008.

[14] R. C. Browning, J. R. Modica, R. Kram, and A. Goswami, "The effects of adding mass to the legs on the energetics and biomechanics of walking," Medicine \& Science in Sports \& Exercise, vol. 39, no. 3, pp. 515-525, 2007.

[15] J. Zhang, C. C. Cheah, and S. H. Collins, "Experimental comparison of torque control methods on an ankle exoskeleton during human walking," in Proceedings of the 2015 IEEE International Conference on Robotics and Automation, ICRA 2015, pp. 55845589, USA, May 2015.

[16] J. M. Brockway, "Derivation of formulae used to calculate energy expenditure in man," Hum Nutr Clin Nutr, vol. 41, no. 6, pp. 463-471, 1987.

[17] M. F. Eilenberg, H. Geyer, and H. Herr, "Control of a powered ankle-foot prosthesis based on a neuromuscular model," IEEE Transactions on Neural Systems and Rehabilitation Engineering, vol. 18, no. 2, pp. 164-173, 2010.

[18] J. Markowitz, P. Krishnaswamy, M. F. Eilenberg, K. Endo, C. B. Chris, and H. Herr, "Speed adaptation in a powered transtibial prosthesis controlled with a neuromuscular model," Philosophical Transactions of the Royal Society B: Biological Sciences, vol. 366, no. 1570, pp. 1621-1631, 2011. 
[19] J. Markowitz and H. Herr, "Human Leg Model Predicts Muscle Forces, States, and Energetics during Walking," PLoS Computational Biology, vol. 12, no. 5, Article ID e1004912, 2016.

[20] P. Krishnaswamy, E. N. Brown, and H. M. Herr, "Human leg model predicts ankle muscle-tendon morphology, state, roles and energetics in walking," PLoS Computational Biology, vol. 7, no. 3, Article ID e1001107, 2011.

[21] J. He, W. S. Levine, and G. E. Loeb, "Feedback Gains for Correcting Small Perturbations to Standing Posture," IEEE Transactions on Automatic Control, vol. 36, no. 3, pp. 322-332, 1991.

[22] C. C. Raasch, F. E. Zajac, B. Ma, and W. S. Levine, "Muscle coordination of maximum-speed pedaling," Journal of Biomechanics, vol. 30, no. 6, pp. 595-602, 1997.

[23] M. Palmer, Sagittal Plane Characterization of Normal Human Ankle Function Across a Range of Walking Gait Speeds, Masters Thesis [Masters, thesis], MIT, 2002.

[24] R. Margaria, Biomechanics and Energetics of Muscular Exercise, Clarendon Press, Oxford, 1976.

[25] P.-C. Kao, C. L. Lewis, and D. P. Ferris, "Invariant ankle moment patterns when walking with and without a robotic ankle exoskeleton," Journal of Biomechanics, vol. 43, no. 2, pp. 203-209, 2010.

[26] C. L. Lewis and D. P. Ferris, "Erratum to "Invariant hip moment pattern while walking with a robotic hip exoskeleton" [J. Biomech. 44 (5) (2011) 789-793]," Journal of Biomechanics, vol. 47, no. 7, p. 1748, 2014.

[27] T. M. Griffin, T. J. Roberts, and R. Kram, "Metabolic cost of generating muscular force in human walking: insights from loadcarrying and speed experiments," Journal of Applied Physiology, vol. 95, no. 1, pp. 172-183, 2003.

[28] R. R. Neptune, S. A. Kautz, and F. E. Zajac, "Contributions of the individual ankle plantar flexors to support, forward progression and swing initiation during walking," Journal of Biomechanics, vol. 34, no. 11, pp. 1387-1398, 2001.

[29] R. R. Neptune, F. E. Zajac, and S. A. Kautz, "Muscle force redistributes segmental power for body progression during walking," Gait \& Posture, vol. 19, no. 2, pp. 194-205, 2004.

[30] J. M. Caputo and S. H. Collins, "An experimental robotic testbed for accelerated development of ankle prostheses," in Proceedings of the IEEE International Conference on Robotics and Automation (ICRA '13), pp. 2645-2650, Karlsruhe, Germany, May 2013.

[31] Y. Ding, I. Galiana, A. T. Asbeck et al., "Biomechanical and physiological evaluation of multi-joint assistance with soft exosuits," IEEE Transactions on Neural Systems and Rehabilitation Engineering, vol. 25, no. 2, pp. 119-130, 2017.

[32] L. M. Mooney and H. M. Herr, "Biomechanical walking mechanisms underlying the metabolic reduction caused by an autonomous exoskeleton," Journal of NeuroEngineering and Rehabilitation, vol. 13, no. 1, article no. 111, 2016.

[33] R. W. Jackson and S. H. Collins, "An experimental comparison of the relative benefits of work and torque assistance in ankle exoskeletons," Journal of Applied Physiology, vol. 119, no. 5, pp. 541-557, 2015.

[34] J. Perry, Gait Analysis: Normal and Pathological Function, SLACK, Inc, New Jersey, NJ, USA, 1992.

[35] E. M. Arnold, S. R. Hamner, A. Seth, M. Millard, and S. L. Delp, "How muscle fiber lengths and velocities affect muscle force generation as humans walk and run at different speeds," Journal of Experimental Biology, vol. 216, no. 11, pp. 2150-2160, 2013.
[36] A. V. Hill, "The heat of shortening and the dynamic constants of muscle," Proceedings of the Royal Society B, vol. 126, no. 843, pp. 136-195, 1938.

[37] A. V. Hill, "The efficiency of mechanical power development during muscular shortening and its relation to load," Proceedings of the Royal Society B Biological Science, vol. 159, no. 975, pp. 319-324, 1964.

[38] K. Endo and H. Herr, "A model of muscle-tendon function in human walking," in Proceedings of the 2009 IEEE International Conference on Robotics and Automation, ICRA '09, pp. 19091915, Japan, May 2009.

[39] H. Geyer, A. Seyfarth, and R. Blickhan, "Positive force feedback in bouncing gaits?" Proceedings of the Royal Society B Biological Science, vol. 270, no. 1529, pp. 2173-2183, 2003.

[40] R. B. Stein and C. Capaday, "The modulation of human reflexes during functional motor tasks," Trends in Neurosciences, vol. 11, no. 7, pp. 328-332, 1988.

[41] M. Knikou and W. Rymer, "Erratum: effects of changes in hip joint angle on H-reflex excitability in humans," Experimental Brain Research, vol. 143, no. 4, pp. 149-159, 2002. 


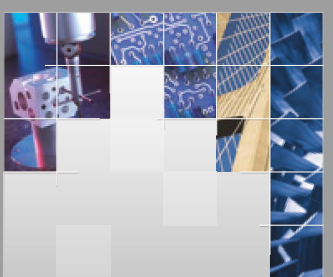

\section{Enfincering}
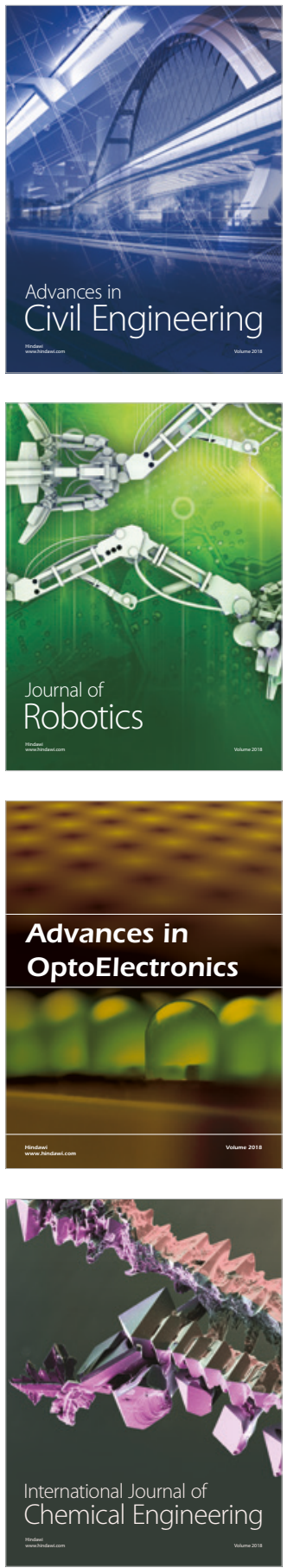

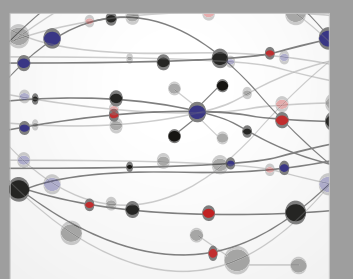

\section{Rotating \\ Machinery}

The Scientific World Journal

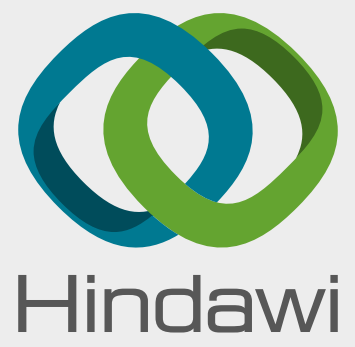

Submit your manuscripts at

www.hindawi.com
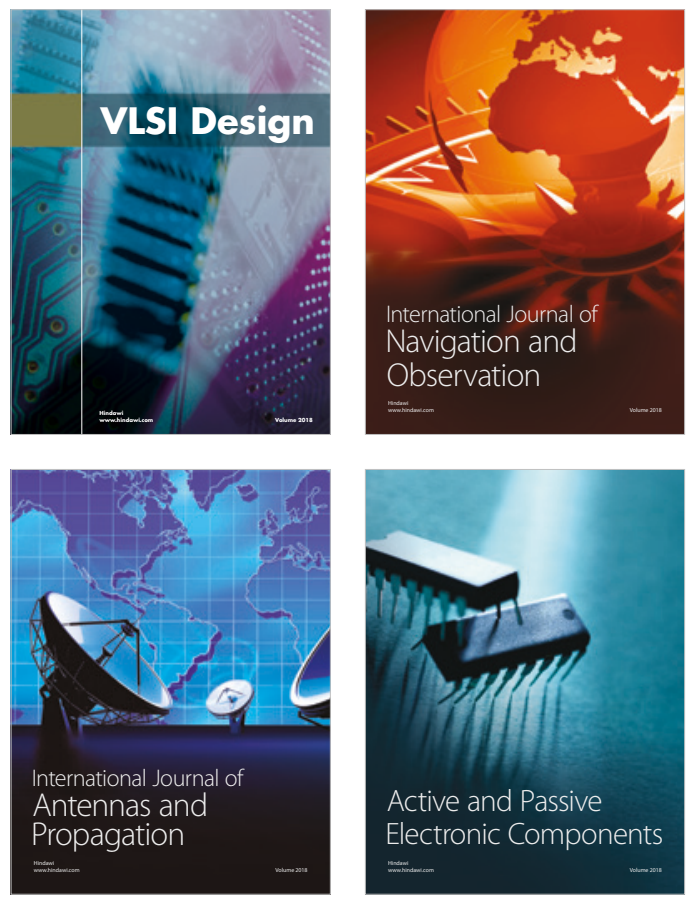
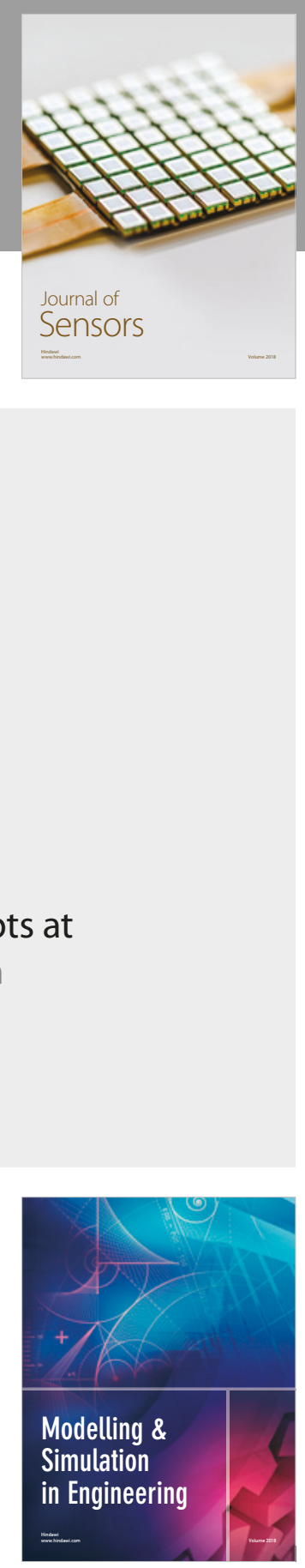

\section{Advances \\ Multimedia}
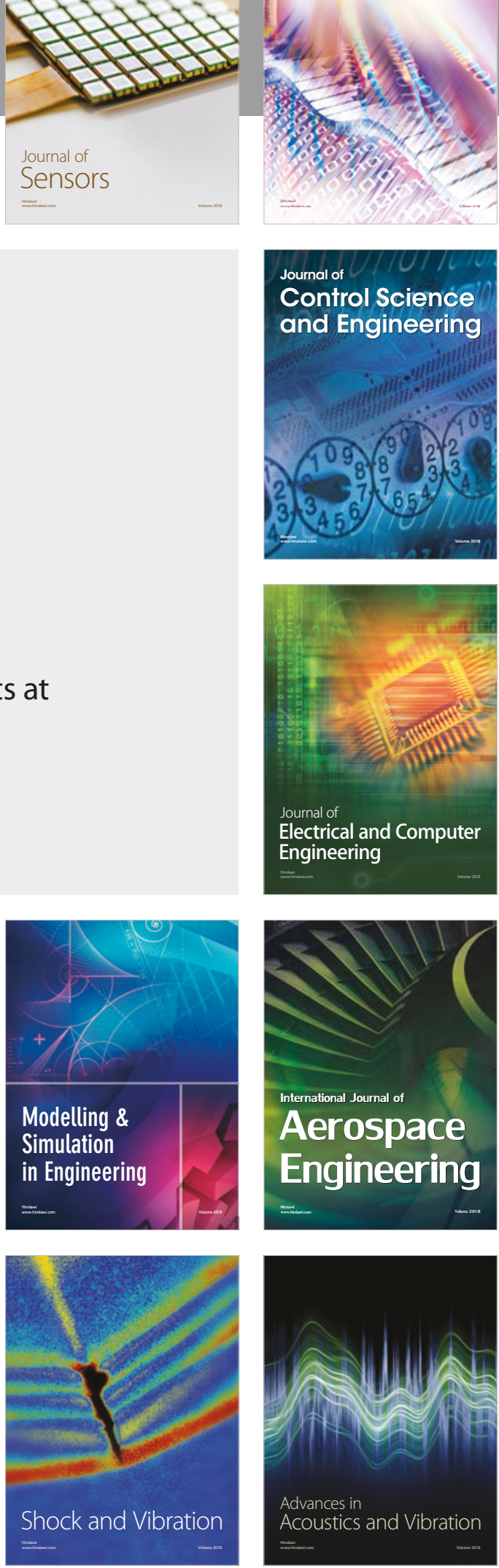